\title{
Novel Chinese pseudorabies virus variants undergo extensive recombination and rapid interspecies transmission
}

\author{
Jinbiao Liu ${ }^{1}$, Changhai Chen ${ }^{1}$, and Xiangdong $\mathrm{Li}^{2}$ \\ ${ }^{1}$ Affiliation not available \\ ${ }^{2}$ Yangzhou University
}

August 5, 2020

\begin{abstract}
Chinese PRV variants have frequent recombination and preferential interspecies transmission. So far, 23 confirmed PRV-infected human cases have been reported. In this letter, we discussed the significance of PRV recombination and its public health issue as an occupational zoonosis.
\end{abstract}

\section{Novel Chinese pseudorabies virus variants undergo extensive recombination} and rapid interspecies transmission

Jinbiao Liu ${ }^{1,2}$, Changhai Chen ${ }^{3}$, Xiangdong $\mathrm{Li}^{1,2}$

${ }^{1}$ Jiangsu Co-innovation Center for Prevention and Control of Important Animal Infectious Diseases and Zoonoses, College of Veterinary Medicine, Yangzhou University, Yangzhou, 225009 China.

${ }^{2}$ Joint International Research Laboratory of Agriculture and Agri-Product Safety, the Ministry of Education of China, Yangzhou University, Yangzhou, 225009 China.

${ }^{3}$ Jiangsu Provincial Center for Animal Disease Control and Prevention, Nanjing, Jiangsu 210036, China.

Correspondence : Prof. Xiangdong Li, Email: 007352@yzu.edu.cn

Dear Editor,

We have some comments related to the recently published paper in Transboundary and Emerging Diseases titled "Genetic evolution analysis of novel recombinant pseudorabies virus strain in Sichuan, China" (Huang et al., 2020). A Chinese pseudorabies virus (PRV) isolate named FJ62 was reported to be a natural recombination of PRV genotype 1 from wild boar and genotype 2 from domestic pigs. Specifically, the gB gene of PRV FJ62 shared the highest homology $(100 \%)$ with Japanese MY-1 strain from wild boar, which shared the same nucleotide sequences with European and American PRV strains. By contrast, gC, gD and gE genes of PRV FJ62 were closely related to Chinese PRV variants in domestic pigs that were far removed from the reference strains in Europe and USA.

Genetically, PRVs could be classified into two genotypes based on genomic sequence or viral gC gene. PRV strains in China belong to genotype 2, whereas PRV isolates from other countries belong to genotype 1. Within genotype 2, Chinese PRV isolates are further divided into two subgenotypes: classical PRV (PRV isolates before 2011) in subgenotype 1 and PRV variants isolated since 2011 in subgenotype 2. As a large DNA virus, the mutation and recombination rates of PRV are theoretically slow. However, frequent intergenotype and intra-genotype recombinations have been reported. In a recent study, He et al (2019) reported that 29 PRVs had undergone recombination, with more inter- than intra-genotype recombinations found. 
The mutation and recombination of viral glycoproteins - such as $\mathrm{gB}, \mathrm{gC}, \mathrm{gD}$ and some other undefined protective immunity-elicited proteins - might partially explain the incomplete protection provided by PRV vaccine strains.

Besides frequent recombination, Chinese PRV variants undergo preferential interspecies transmission including cows, dogs, cats, sheep, minks, foxes, racoons, and even humans (Wong et al., 2019, Li et al., 2020). PRV utilizes its glycoprotein gD to bind to cells via the nectin-1 receptor, which shares conserved amino acid residues among different species (Li et al., 2017). Zheng et al recently reported a human severe pseudorabies encephalitis in Transboundary and Emerging Diseases (Zheng et al., 2019). Besides that, another 22 PRV variants-infected human cases have been reported (Table 1). There are several distinct characteristics of patients infected with PRV variants versus those infected with classical PRV. First, humans infected with classical PRV had fever, weakness and pruritus before progressing to neurological signs, including dysphagia and paresthesia. The clinical signs of patients can last several months, but all eventually recovered without any treatment. By contrast, humans infected with PRV variants suffered severe central nervous system disorders and fatal encephalitis. Besides encephalitis, 12 out of 23 patients also suffered from endophthalmitis, causing loss of vision. How PRV variants invade the ocular nerve system and leads to endophthalmitis warrants further investigation. Second, humans infected with classical PRVs had close contact with diseased dogs, cats or cattle (Wong et al., 2019). By contrast, all patients infected with PRV variants had close contact with pigs. This suggested that pigs have become the etiological source of PRV for human infection. Third, PRV variants have been successfully isolated on cells from cerebrospinal fluid samples of patients. Most recently, human PRV hSD-1/2019 strain was, for the first time, isolated from cerebrospinal fluid samples on PK-15 cells (Liu et al., 2020). The virus was shown to be a PRV variant by next-generation sequencing; it induced acute neurological signs in pigs, and caused high mortality.

The above reported cases suggest that PRV could be an important neglected occupational zoonotic pathogen in humans. Therefore, PRV should be screened for in unexplained fever and encephalitis cases in human, especially when patients have a history of close contact with pigs or pig products. The mechanisms by which PRV variants lead to encephalitis and endophthalmitis should be further explored in non-human primate models.

\section{ACKNOWLEDGEMENTS}

The authors were supported by Development of Jiangsu Higher Education Institutions (PADD) and Tenthousand Talents Program, and grants from Jiangsu Co-innovation Center for Prevention and Control of Important Animal Infectious Diseases and Zoonoses.

\section{CONFLICT OF INTEREST}

The author declares no conflict of interest relevant to this article.

\section{ETHICAL APPROAL}

Not applicable to this comment.

\section{Data Availability Statement}

There is no experimental data available in this letter.

\section{REFERENCES}

Huang, J., L. Zhu, J. Zhao, X. Yin, Y. Feng, X. Wang, X. Sun, Y. Zhou and Z. Xu, 2020: Genetic evolution analysis of novel recombinant pseudorabies virus strain in Sichuan, China. Transboundary and emerging diseases, 67, 1428-1432.

Li, A., G. Lu, J. Qi, L. Wu, K. Tian, T. Luo, Y. Shi, J. Yan and G. F. Gao, 2017: Structural basis of nectin-1 recognition by pseudorabies virus glycoprotein D. PLoS pathogens,13, e1006314. 
Li, X. D., S. H. Fu, L. Y. Chen, F. Li, J. H. Deng, X. C. Lu, H. Y. Wang and K. G. Tian, 2020: Detection of Pseudorabies Virus Antibodies in Human Encephalitis Cases.Biomedical and environmental sciences : BES, $33,444-447$.

Liu, Q., X. Wang, C. Xie, S. Ding, H. Yang, S. Guo, J. Li, L. Qin, F. Ban, D. Wang, C. Wang, L. Feng, H. Ma, B. Wu, L. Zhang, C. Dong, L. Xing, J. Zhang, H. Chen, R. Yan and W. Li, 2020: A novel human acute encephalitis caused by pseudorabies virus variant strain. Clinical infectious diseases, doi: $10.1093 / \mathrm{cid} /$ ciaa987.

Wong, G., J. Lu, W. Zhang and G. F. Gao, 2019: Pseudorabies virus: a neglected zoonotic pathogen in humans? Emerging microbes $\mathcal{E}$ infections, 8, 150-154.

Zheng, L., X. Liu, D. Yuan, R. Li, J. Lu, X. Li, K. Tian and E. Dai, 2019: Dynamic cerebrospinal fluid analyses of severe pseudorabies encephalitis. Transboundary and emerging diseases, 66, 2562-2565.

Table 1 . Summary of reported human PRV cases.

\begin{tabular}{|c|c|c|c|c|c|c|c|c|}
\hline$\overline{\text { Case }}$ & Year & Sex & Age & Province & Occupation & Clinical signs & Clinical signs & Outcome \\
\hline & & & & & & Encephalitis & Endophthalmitis & \\
\hline 1 & 2018 & Male & 25 & Hubei & Veterinary & Yes & Yes & Survived \\
\hline 2 & 2019 & Male & 35 & Henan & Pig butcher & Yes & No & Survived \\
\hline 3 & 2019 & Male & 49 & Henan & Pig butcher & Yes & No & Survived \\
\hline 4 & 2018 & Male & 43 & Shandong & Veterinary & Yes & No & Survived \\
\hline 5 & 2018 & Male & Mid-aged & Shandong & Butcher & Yes & No & Died \\
\hline 6 & 2018 & Male & Mid-aged & Hebei & Swineherd & Yes & Yes & Survived \\
\hline 7 & 2018 & Male & Young & Guangdong & Driver & Yes & Yes & Survived \\
\hline 8 & 2011 & Female & Young & Beijing & Pork dealer & Yes & No & Died \\
\hline 9 & 2017 & Male & 55 & Inner Mongolia & Pork dealer & Yes & Yes & Died \\
\hline 10 & 2017 & Male & 51 & Inner Mongolia & Cook & Yes & No & Died \\
\hline 11 & 2017 & Male & 38 & Shandong & Butcher & Yes & No & Survived \\
\hline 12 & 2016 & Female & 42 & Hebei & Unknown & Yes & Yes & Survived \\
\hline 13 & 2019 & Male & 44 & Anhui & Pork dealer & Yes & Yes & Survived \\
\hline 14 & 2019 & Male & 44 & Shandong & Pork dealer & Yes & Yes & Survived \\
\hline 15 & 2018 & Male & 50 & Shandong & Pig slaughterer & Yes & No & Survived \\
\hline 16 & 2018 & Female & 50 & Shandong & Pork cutter & Yes & No & Survived \\
\hline 17 & 2018 & Male & 43 & Shandong & Pig handler & Yes & Yes & Survived \\
\hline 18 & 2018 & Male & 59 & Shandong & Pork cutter & Yes & No & Survived \\
\hline 19 & 2018 & Male & 50 & Shandong & Pork cutter & Yes & Yes & Survived \\
\hline 20 & 2018 & Male & 59 & Hebei & Swineherd & Yes & Yes & Survived \\
\hline 21 & 2019 & Male & 43 & Shandong & Veterinary & Yes & No & Survived \\
\hline 22 & 2019 & Male & 49 & Anhui & Pig slaughterer & Yes & Yes & Survived \\
\hline 23 & 2017 & Female & 46 & Jiangxi & Swineherd & Yes & Yes & Survived \\
\hline
\end{tabular}

